\title{
On the Accuracy of Weighted Proximity Based Localization in Wireless Sensor Networks
}

\author{
Peter Brida ${ }^{1}$, Jan Duha ${ }^{1}$, Marek Krasnovsky ${ }^{1}$ \\ ${ }^{1}$ University of Zilina, Faculty of Electrical Engineering, \\ Department of Telecommunications, \\ Univerzitna 1, 01026 Zilina, Slovakia \\ \{Peter.Brida, Jan.Duha, Marek.Krasnovsky\}@fel.uniza.sk
}

\begin{abstract}
Localization positioning is one of the basic problems in wide wireless sensor networks. The main objective of positioning process is to define the location of a sensor (node or device) from the relevant information obtained from reference nodes. These nodes already know their location. In many cases, these reference nodes are called beacons. The paper deals with proximity based location technique and their modifications. It does not belong to the most accurate techniques, but on the other hand it is low cost alternative to more expensive techniques. The objective of the paper is to give a survey of various proximity based location techniques performance. We analyze the influence of various design choices on the accuracy of localization techniques. The results of basic proximity based localization techniques are compared with modified proximity technique, which is proposed in this paper.
\end{abstract}

Keywords: Proximity based localization, localization error, weighted proximity based localization, wireless ad hoc sensor networks.

\section{Introduction}

In the near future, our surrounding will consist of large networks of wireless sensor nodes. Particularly, applications like environmental monitoring of water and soil require that these nodes be very small, light and unobtrusive. A wireless ad hoc sensor network (WASN) consists of a possibly large number of wireless devices is able to take environmental measurements (e.g. temperature, light, sound, and humidity). These sensor readings are transmitted over a wireless channel to a running application that makes decisions based on these sensor readings. Many applications have been proposed for wireless sensor networks, and many of these applications have specific quality of service (QoS) requirements that offer additional challenges to the application designers. Several applications have been envisioned for wireless sensor networks [1]. These range in scope from military applications to environment monitoring to biomedical applications. 


\section{Mobile Localization in Wireless Networks}

The concept of localization is not limited to the geographic representation of physical location with sets of coordinates (latitude, longitude, and altitude). It is also applicable to symbolic location in a non-geographic sense such as location in time or in a virtual information space such as a data structure or the graph of a network.

Common to all notions of location is the concept that the individual locations are all relative to each other, meaning that they depend on a predefined frame of reference. This leads to a differentiation of the relative and absolute positioning [2].

When position information is used in reference to a geographic map or a global time reference, context information can be extended. An absolute position is given in respect to an inertial system and a reference point in this inertial system (see Fig. 1 a). On the other side, a relative position can only be given in respect to other points resolving the distances and the geometric configuration, e.g., the topology (see Fig. 1 b).

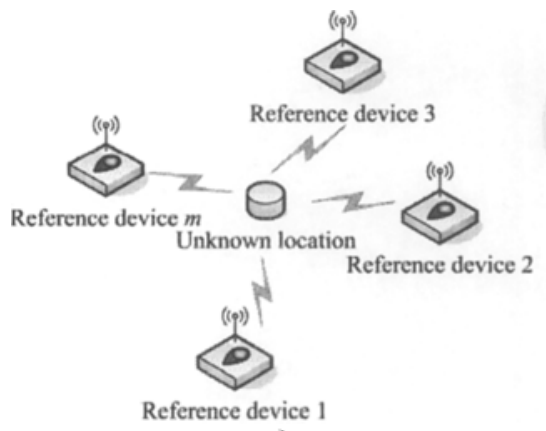

a)

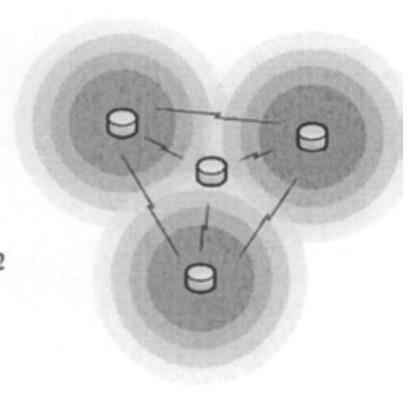

b)

Fig. 1. a) Absolute positioning

b) Relative positioning.

When talking about physical location in the traditional way, points are usually viewed as three-dimensional coordinates $[x, y, z]$ in a Cartesian reference coordinate system.

Usually, $[x, y, z]$ coordinates by themselves are not meaningful for context-aware system services and other information needs to be associated with these position fixes. In these cases, it is important to introduce the fourth dimension - time to be able to specify where and when a certain event took place resulting in sets of $[x, y, z, t]$ for each position fix. This four-dimensional fix can then be used to put subsequent events into a context frame.

Localization techniques generally consist of three components [2]:

- Identification and data exchange.

- Measurement and data acquisition.

- Computation to derive location.

The various approaches divide these tasks differently across their system components and the data exchange between nodes is used in some cases no, or only unidirectional. 
The mechanisms used for estimating location are possible to divide into two categories: range-based and range-free [3]. The range-based mechanisms are based on the indirect measurements of distance or angle between sensors. Examples of the mechanism are the following methods: received signal strength indicator (RSSI), time of arrival (ToA), time difference of arrival (TDoA), carrier phase and code measurements, ultra wide-band (UWB), ultrasound, and even visible light pulses or the angle of arrival (AoA) of a radio signal. The important thing to note is that these mentioned measurements always have errors and individual measurements are not independent of each other and are strongly influenced by the surrounding environment and the used transmission system. Because of the hardware limitations of WASN devices, solutions in range-free localization are being pursued as a costeffective alternative to more expensive range-based approaches.

\subsection{Localization in Wireless Ad hoc Sensor Networks}

Wireless Ad hoc Sensor Networks (WASNs) are the special part of wireless Ad hoc networks. The localization in WASNs is a little bit different in comparison with localization in traditional wireless networks (e.g. cellular networks).

WASN is usually characterized by subjects such as vast number of devices (nodes), high overall node mobility, considerable power and resource consumption at the nodes, and moderate network sizes. WASN have brought about quite a change in the traditional connection-oriented infrastructure-dominated telecommunications world [2]. Unpredictable dynamics due to failures and changes in nodes, the environment as well as deployment in uncontrolled areas with high dynamics and possibly hostile to radio signal propagation require adaptable networking mechanisms. Other characteristics of typical WASN nodes are the limited resources available on these low power embedded systems, especially the limited transmission range and low duty cycle operation of the radio transceivers. Nodes can be reactive and are able to wake up on demand [2]. Targeted for a very long lifespan, integrated into all kinds of everyday objects and building materials, deployed once, and in many cases never collected again or decommissioned, the vast majority of nodes will form a quasistatic, multihop network topology.

The problem of localization, i.e., the determination, where a given node is physically located in a network, is a challenging one, and yet extremely crucial for many of these applications. Practical considerations such as the small size, form factor, cost and power constraints of nodes preclude the reliance on Global Positioning System (GPS) in all nodes of these networks.

\subsection{Proximity Based Localization}

Proximity based localization belongs to the group of range-free localization. Localization using proximity measurements is popular, when low cost takes precedence in priority over accuracy. Since, messages necessarily pass between neighbors, there is no additional bandwidth required to proximity. Proximity measurements simply report whether or not two devices are 'connected' or 'in-range'. 
However, the term 'in-range' may mislead readers to believe that proximity is purely a function of geometry - whether or not two devices are separated by less than a particular distance. In fact, proximity is determined by whether or not a receiver can demodulate and decode a packet sent by a transmitter. Given the received signal and noise powers, the successful reception of a packet is a random variable. The proximity carries considerable information regarding sensor location in a binary variable. The proximity based localization has been used by numerous researchers for localization in ad hoc and wireless sensor networks [4], [5], [6] and [7].

A fixed number of reference points in the network with overlapping regions of coverage transmit periodic beacon signals. The nodes use a simple connectivity metric that is more robust to environmental vagaries, to infer proximity to a given subset of these reference points. Devices localize themselves to the centroid of their proximate reference points. The accuracy of localization is then especially dependent on the separation distance between two or more adjacent reference points and the transmission range of these reference points. In this paper we propose a weighted proximity based localization. The utilized influence of particular nodes for location estimation is increased, and on the other side the impact of other nodes is reduced. This simple enhancement leads to increasing of positioning accuracy without significant innovation.

\section{System and Measurement Models}

WASNs are made up of peer-to-peer links between devices (nodes). Pair-wise measurements can be made from any of these links, but only a small fraction of devices have coordinate knowledge. Thus, the measurements are made primarily between pairs of devices of which neither has known coordinates. The device with known coordinates is called a reference device. Otherwise, it is referred to as blindfolded device, since it cannot see their location. Specifically, consider a network of $m$ reference and $n$ blindfolded devices. The relative location problem corresponds to the estimation of blindfolded device coordinates. For simplicity, we consider the location in 2D plane. Let $\left[x_{i} ; y_{i}\right]^{\mathrm{T}} i=1,2, \ldots, m$ are coordinates of reference devices and $\left[x_{j} ; y_{j}\right]^{\mathrm{T}} j=1,2, \ldots, n$ are coordinates of blindfolded devices. Pair-wise measurements $\left\{R S S_{i, j}\right\}$ are done, where $R S S_{i, j}$ is a measurement between devices $i$ and $j . R S S_{i, j}$ is received signal strength in device $j$ from the device $i$.

\subsection{Channel Model}

We consider a fading channel in all measurements in this paper. The propagation path between two devices is shown in fig. 2 , where RSS is received signal strength, $T_{x}$ is transmitted signal strength. The influence signal attenuation is defined by: $L_{L S}$ is signal degradation caused by large-scale propagation, $L_{M S}$ is signal degradation caused by medium-scale propagation and finally $L_{S S}$ is signal degradation caused by small-scale propagation. All these parameters are in [dBm]. Parameters $L_{M S}$ and $L_{S S}$ have the normal distribution, but their impact on the proximity based localization is negligible, because most of devices are static and these parameters are primarily 
changed during motion of device. Therefore, we take into consideration idealized radio model only $L_{L S}$. We chose this model because it is mathematically simple and easy. In this section, this model is presented. We make two assumptions in the model: the perfect spherical radio propagation and identical transmission range (power) for all devices.

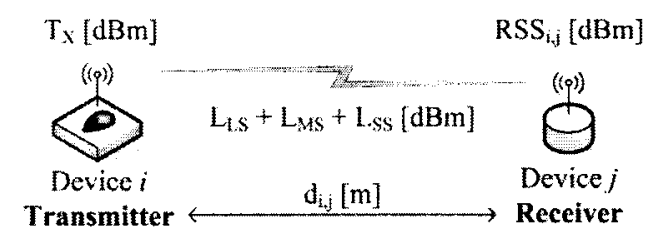

Fig. 2. Channel model.

$R S S_{i, j}$ is the measured received signal strength at device $j$ transmitted by device $i$ (in $\mathrm{dBm}$ ) is Gaussian

$$
R S S_{i, j}(\mathrm{dBm})=P_{0}(\mathrm{dBm})-10 \cdot n_{p} \cdot \log \left(d_{i, j} / d_{0}\right),
$$

where $R S S_{i, j}$ is the mean power in $\mathrm{dBm}, P_{\theta}$ is the received signal strength at the reference distance $d_{0}$. Typically $d_{0}=1$ meter, and $P_{0}$ is calculated by the free space path loss formula [2]. The path loss exponent $n_{p}$ is a function of the environment.

\subsection{Proximity Measurements}

The proximity measurement $S$ is determined based on the measured signal. $S_{i, j}$ is obtained from $R S S_{i, j}$ and it is equal to 1 if devices $i$ and $j$ are in range, and it is 0 if not. It is necessary to clearly define transition from status "in range" to "out of range". Therefore, we define a threshold. If the received signal strength $\left(R S S_{i, j}\right)$ at $j$ device transmitted by $i$ device is higher as defined threshold then $i$ devices is assumed to be in range of the $j$ device. Thus,

$$
S_{i, j}=\left\{\begin{array}{ll}
1, & R S S_{i, j} \geq R S S_{T} \\
0, & R S S_{i, j}<R S S_{T}
\end{array} .\right.
$$

\subsection{Simulation Environment}

The presented numerical simulations compare the performance of various solutions of proximity localization. Simulations are done for one propagation environment. The all devices are situated in square $10 \mathrm{~m} \times 10 \mathrm{~m}$. The reference devices locations are modeled in two cases. In the first case, the reference devices lay in expressly defined point with equally spacing, the reference devices forming raster with equally defined spacing. The raster spacing depends on the number of reference devices used in simulation. In the second case, we assume the reference devices are situated randomly (according to Gaussian distribution $\mathrm{N}(0,1)$ ) in mentioned area. A thousand trials are 
performed. In each trial, the positions of reference devices and blindfolded device are generated at first. In the next step, $R S S$ are calculated between each reference devices and blindfolded device based on the equation (1). Here, we use the parameters $n_{p}=2$ (free space). Then, the devices are rejected which do not fulfil the threshold condition. The location coordinates of blindfolded device are determinated based on the remaining reference devices. The location coordinates can be calculated based on the following techniques (our working titles):

- Common proximity (CMP) - the estimated location is determined on the basis of the closest device location, i.e. the blindfolded device has same location coordinates as the closest device.

$$
\left[x_{\text {_c }} c m p_{\text {est }} ; y_{\ldots} c m p_{\text {est }}\right]=\left[x_{\text {closestdevice }} y_{\text {closestdevice }}\right]
$$

- Centroid proximity (CNP) - the position of blindfolded device is calculated as mean value of coordinates $N$ of the closest reference devices. It is defined by the centroid of these reference devices (see equation (4)).

$$
\left[x_{-} c n p_{e s t} ; y_{-} c n p_{e s t}\right]=\left[\frac{1}{N} \sum_{i=1}^{N} x_{i} ; \frac{1}{N} \sum_{i=1}^{N} y_{i}\right]
$$

- Weighted proximity (WEP) - the principle of this algorithm results from centroid proximity, but the each input part (particular coordinates of devices) is individually weighted. The fundamental of WEP is the increasing of influence of closer reference devices at the expense of further devices. The mean value of coordinates obtained after the weighting gives the WEP estimate. The main benefit of the WEP algorithm is the accuracy gain by combining information contributed from multiple part inputs. Fig. 3 shows the overview of the WEP algorithm to fuse $N$ reference devices (input parts).

$$
\begin{aligned}
& \text { Input part: Weight ! } \\
& \underset{2}{\text { Input part Weight } 2} \sum \sum_{x}^{\text {Locution }} \text { Estimation, }\left[x_{-} \text {wep } p_{n}, y_{-} \text {wep } p_{s s t}\right] \\
& \text { Input pary } \\
& \text { Weight } N
\end{aligned}
$$

Fig. 3. Overview of the weighted proximity (WEP) algorithm.

We are interested in calculate location estimate from $N$ available the closest reference devices, where $\left[x_{j} ; y_{j}\right]^{\mathrm{T}} j=1,2, \ldots, N$ is vector of their coordinates. Then, the WEP estimate $\left.\left[x_{-} w_{e} p_{e s i} ; y_{-} w_{e s}\right]_{e s}\right]^{\mathrm{T}}$ is written as:

$$
\left[x_{-} w_{\text {est }} ; y_{-} w_{e p}\right]=\left[\sum_{i=1}^{N} x_{i} \cdot w_{i} \cdot\left(\sum_{i=1}^{N} w_{i}\right)^{-1} ; \sum_{i=1}^{N} y_{i} \cdot w_{i} \cdot\left(\sum_{i=1}^{N} w_{i}\right)^{-1}\right] \text {, }
$$

where $\left[w_{j}\right]^{\mathrm{T}} j=1,2, \ldots, N$ are input weights. 
The accuracy of device positioning is compared by means of RMSE (Root Mean Square Error)

$$
R M S E=\sqrt{\left(x_{r}-x_{e s t}\right)^{2}+\left(y_{r}-y_{e s t}\right)^{2}}[\mathrm{~m}]
$$

where $\left[x_{r} ; y_{r}\right]$ are coordinates of the real (precise) position and $\left[x_{e s t} ; y_{e s t}\right]$ are coordinates of estimated position on the basis of the selected technique.

\section{Simulation Results}

In this section, we discuss obtained results by means of described techniques above mentioned. The results are simulated to evaluate the performance of the proximity localization in WASNs. The simulations are realized for each allocation of reference devices mentioned in previous part as following criterions:

- Impact of observed weights on the location accuracy.

- Influence of reference devices allocation on the location accuracy.

- Optimalization of number reference devices used for location estimation in all proximity techniques.

- Influence of number all reference devices used in simulation of all proximity techniques.

In the first experiment, an influence of four different weights used for weighting particular input data is examined, i.e. coordinates of reference devices. Only the three closest reference devices are weighted. It results from our preprocessing, because the accuracy does not significantly increase as the number of weighted reference devices increases.
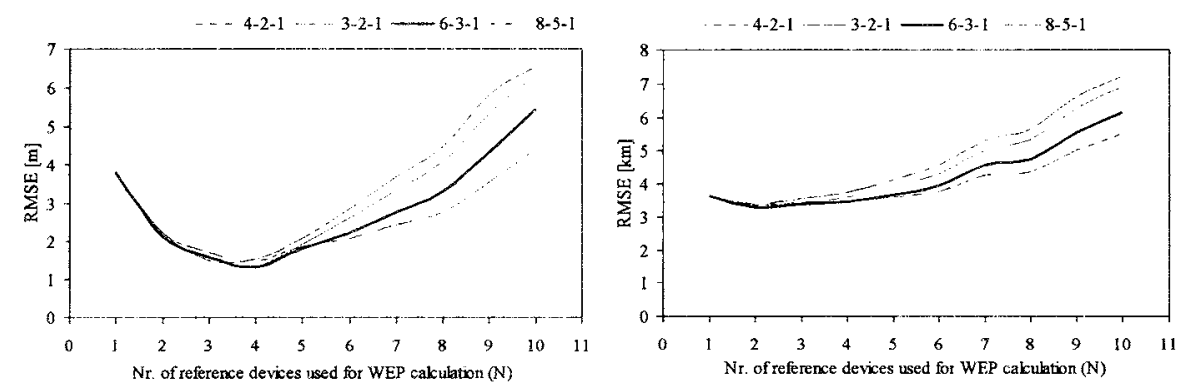

Fig. 4. RMSE $[\mathrm{m}]$ versus the number of reference devices used for WEP calculation $(m=10)$.

Fig 4 is plotted for RMSE properties for $m=10$. It can be seen two different situations from this picture: the reference devices form raster with equally defined spacing on the left and dependency random allocated reference devices on the right. The same weights are observed for both cases, but the different results are achieved. It is caused by the different allocation of reference devices. The most accurate results 
are drawn with bold line in all figures. Of course, the much more accurate results are achieved in the first case (reference devices form raster) in comparison with random allocation of reference devices. The most accurate results are achieved for weight $\{6-$ 3-1 $\}$ in both cases. The minimal localization error (RMSE) is in the case of $N=4$ (the first case). In the second case, the minimal error is for $N=2$. These numbers are important for next processing in particular reference devices allocation.

The above simulation is repeated for the case of $m=100$.
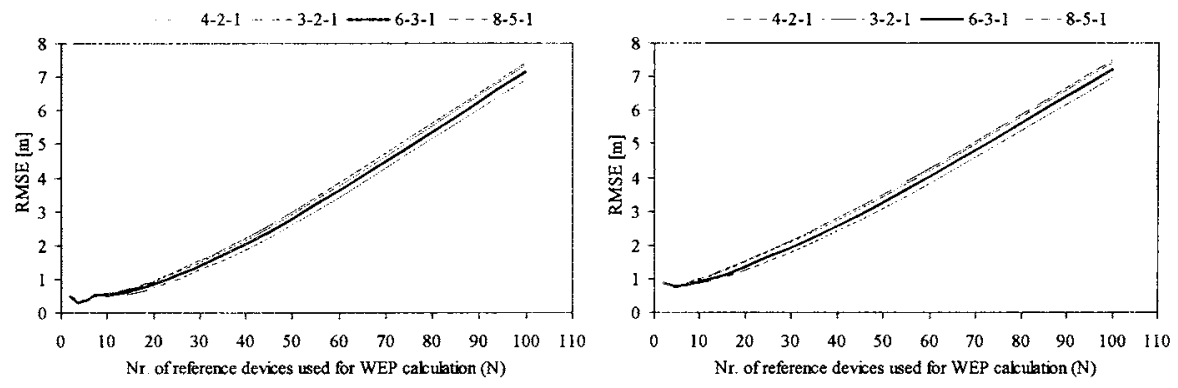

Fig. 5. RMSE [m] versus the number of reference devices used for WEP calculation $(m=100)$.

Fig. 5 depicts RMSE properties for 100 reference devices $(m=100)$. It can be seen two different situations from this picture: the reference devices form raster with equally defined spacing on the left and dependency random allocated reference devices on the right. In both cases, the same weights are observed as well as in previous situation $(m=10)$. We achieved again the different results. Of course, the much more accurate results are achieved in the first case (reference devices form raster) in comparison with random allocation of reference devices. The most accurate results are achieved for weight $\{6-3-1\}$ in both cases. The minimal localization error (RMSE) is in the case of $N=4$ (the first case). In the second case (random allocation), the minimal error is obtained for $N=5$.

The best results are achieved for $m=4$ for the reference devices form raster scenario. It is caused by regular allocation of the reference devices. In this case, the blindfolded device is in the square and four reference devices lay in the square edge. In the following experiments, we use the weight with the best results, e.g. $\{6-3-1\}$.

In the next part, we compare the influence of allocation of reference devices on location accuracy for all observed proximity techniques (CMP, CNP and WEP) just for $m=10$. Because, the results are very similar and more manifestly in comparison with $m=100$.

Fig. 6 shows the dependency of localization error on $N$ (the number of reference devices used for calculation of CNP and WEP location estimation). Naturally, the change of $N$ does not impact on accuracy of common proximity (dotted line). The results of CNP and WEP are similar. The number of reference devices used for estimation calculation plays important role in localization accuracy. The minimal error is interesting for us. The results confirm the fact, that weighted proximity achieves the smallest localization error in comparison of other proximity based techniques. The difference of minimal localization error is the most important factor 
of objectively comparison for observed techniques. In the first case, the difference is not small for $N=4$. Therefore, WEP technique is suitable to use for estimation of location. In next simulation, only four reference devices are used for calculation of device coordinates in CNP and WEP techniques. The situation is different in the second case (random allocated reference devices). In general, we can say that the accuracy decreases as the number of reference devices used for CNP and WEP calculation (except $N=2$ ) increases. The differences are smaller in comparison with the first case, but WEP is still the most accurate technique.
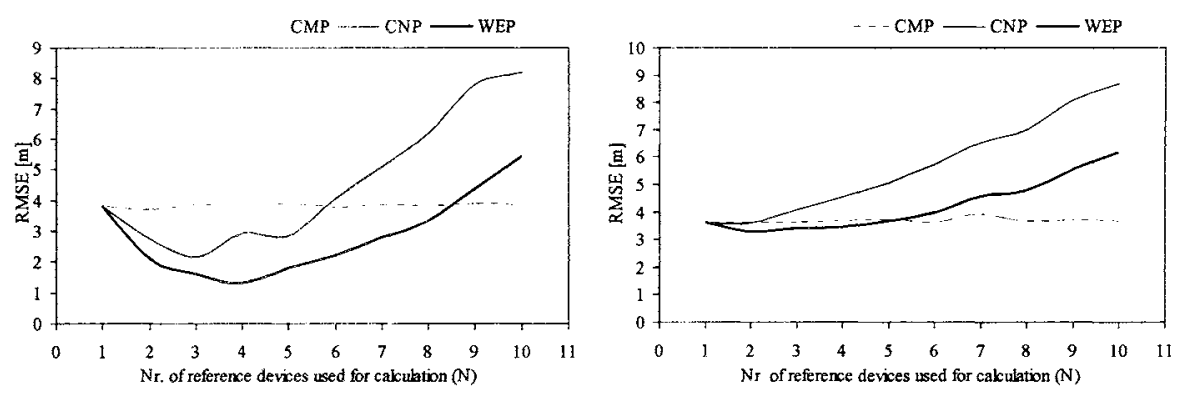

Fig. 6. RMSE $[\mathrm{m}]$ versus $N$ for different allocation of reference devices.

The situation when reference devices lay in the raster is only modeled situation. Hence, we can say that the second case is more similar to reality. The positioning in the first case is much more accurate in comparison to random allocation of the reference devices. Hence, it is necessary to consider about allocation of sensors in process of implementation of WASN.

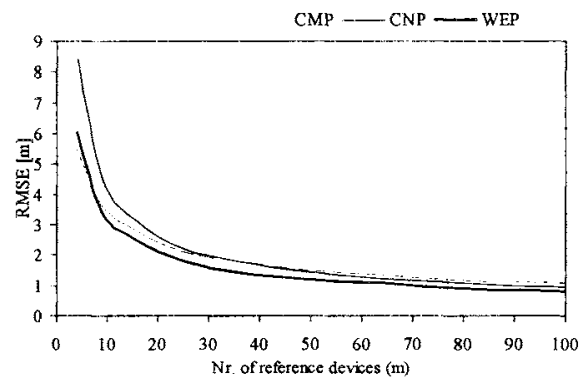

Fig. 7. RMSE $[\mathrm{m}]$ versus the number of reference devices.

Fig. 7 shows the dependence of RMSE on the number of reference devices $m$. On a basis of the obtained results we conclude that the weighted proximity technique achieves more accurate results comparing to centroid and common proximity. The RMSE is an exponential function of the number of reference devices $m$. Ascending value of the reference devices number means increasing of the RMSE. 


\section{Conclusions}

We discussed three techniques for the location determination based on the proximity technology for wireless ad hoc sensor networks. The distinguished advantage of this localization technique is its simplicity and the ease of implementation. In our extensive study, we analyzed the impact of following parameters, i.e. allocation of reference devices, the number of reference devices used for location estimation and the number of all present reference devices in the observed area. The mentioned parameters were tested by means of the common, centroid and weighted proximity based localization technique. According to the results, the performance of the weighted proximity technique is better in comparison with the centroid proximity. The common proximity achieved the worst results, but this variant does not need any calculation capacity for the localization procedure. According to the results, the proposed and verified weight $\{6-3-1\}$ is the most suitable for proximity based localization in wireless ad hoc sensor networks. From this study, the proximity based methods is not accurate in comparison with the sophisticated localization methods, but it is sufficient for certain non-critical applications in huge WASNs.

Acknowledgments. The work on this paper was supported by the grant VEGA 1/4065/07 of Scientific Grant Agency of the Slovak Republic.

\section{References}

1. Stojmenovic, I.: Handbook of sensor networks: algorithms and architectures, A John Wiley \& Sons, 552 pages, ISBN: 978-0-471-68472-5, (2005)

2. Mohammad llyas, Imad Mahgoub: Handbook of sensor networks: compact wireless and wired sensing systems, CRC Press, 672 pages, ISBN 0-8493-1968-4, (2004)

3. Jayashree, L.S., Arumugam, S., Anusha, M., Hariny, A. B.: On the Accuracy of Centroid based Multilateration Procedure for Location Discovery in Wireless Sensor Networks, In proc. Wireless and Optical Communications Networks, (2006)

4. Sundaram, N., Ramanathan, P.: Connectivity based location estimation scheme for wireless ad hoc networks. In IEEE Globecom 2002, volume 1, pages 143-147, (2002)

5. Doherty, L., Pister, K. S. Jo, Ghaoui, L. E.: Convex position estimation in wireless sensor networks. In IEEE Infocom, volume 3, pages 1655-1663, (2001)

6. Nagpal, R., Shrobe, H., Bachrach, J.: Organizing a global coordinate system from local information on an ad hoc sensor network. In 2nd Intl. Workshop on Inform. Proc. In Sensor Networks, (2003)

7. Niculescu, D., Nath, B.: Ad hoc positioning system. In IEEE Globecom 2001, volume 5, pages $2926-2931,(2001)$

8. Patwari, N., Alfred O. Hero III: Using Proximity and Quantized RSS for Sensor Localization in Wireless Networks, In WSNA'03, San Diego, California, USA (2003)

9. Capkun, S., Hamdi, M., Hubaux, J.-P.: GPS-free positioning in mobile ad-hoc networks. In 34th IEEE Hawaii Int. Conf. on System Sciences, (2001) 